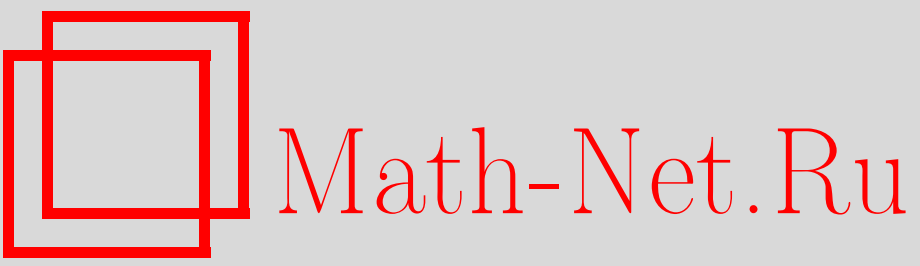

А. И. Бугрий, В.Н.Шадура, Асимптотика корреляционной функции твистованных полей в двумерной модели Дирака на решетке, ТМФ, 1999, том 121, номер 2, 329-346

DOI: https://doi.org/10.4213/tmf813

Использование Общероссийского математического портала Math-Net.Ru подразумевает, что вы прочитали и согласны с пользовательским соглашением

http://www . mathnet.ru/rus/agreement

Параметры загрузки:

IP : 3.81 .55 .215

26 апреля 2023 г., 12:53:55 
ТЕОРЕТИЧЕСКАЯ

И МАТЕМАТИЧЕСКАЯ

ФИЗИКА

Том 121, № 2

ноябрь, 1999

(C) 1999 г.

А.И. Бугрий* , В.Н. Шадура*

\section{АСИМПТОТИКА КОРРЕЛЯЦИОННОЙ ФУНКЦИИ ТВИСТОВАННЫХ ПОЛЕЙ В ДВУМЕРНОЙ МОДЕЛИ ДИРАКА НА РЕШЕТКЕ}

Получено детерминантное представление для корреляционной функции твистованных полей в двумерной теории Дирака на решетке. Эти поля определяются твистованными граничными условиями для дираковских фермионов. Вычислена асимптотика корреляционной функции на больших расстояниях (вакуумное ожидание твистованного поля) в критической точке и в скейлинговой области.

\section{1. ВВЕДЕНИЕ}

В последнее время был достигнут определенный прогресс в вычислении асимптотического поведения корреляционных функций локальных полей в некоторых точнорешаемых моделях двумерных квантовых теорий поля $[1,2]$. Обычно корреляционная функция некоторого локального поля в таких моделях может быть представлена в виде бесконечного ряда формфакторных вкладов, которые вычисляются с использованием идеи формфакторного бутстрапа [3] или метода углового квантования [4, 5]. В работах [6-8] разработана техника суммирования формфакторного разложения, позволяющая получать представление корреляционной функции через детерминанты Фредгольма определенных интегральных операторов. Такое представление позволяет провести анализ асимптотического поведения корреляционной функции.

В данной работе с использованием метода функционального интеграла в двумерной теории дираковского поля на решетке получено детерминантное представление корреляционной функции твистованных полей $\mu_{\nu}(r)$ в фазе беспорядка. При $\nu=1 / 2$ такие поля совпадают с полями беспорядка $\mu(r)$ в этой модели и их корреляционная функция удовлетворяет следующему соотношению $[9,10]$ :

$$
\langle\mu(0) \mu(r)\rangle=\left\langle\mu^{\mathrm{I}}(0) \mu^{\mathrm{I}}(r)\right\rangle^{2},
$$

где $\mu^{\mathrm{I}}(r)$ - поле беспорядка в модели Изинга.

${ }^{*}$ Институт теоретической физики им. Н. Н. Боголюбова НАН Украины, Киев, Украина. E-mail: abugrij@bitp.kiev.ua 
Вычисляя асимптотическое поведение корреляционной функции твистованных полей в критической точке и в скейлинговой области в пределе $r \rightarrow \infty$, мы находим вакуумное ожидание $\left\langle\mu_{\nu}(r)\right\rangle$. Индекс $\nu(0<\nu<1)$ задает твистованные граничные условия для дираковских фермионов вдоль линии корреляции. Поскольку мы получаем указанные асимптотики в рамках одного и того же способа регуляризации, то это позволяет исключить неоднозначность в нормировке корреляционных функций и, следовательно, в нормировке вакуумного ожидания. В скейлинговом пределе (квантовая теория массивных дираковских фермионов) эти поля являются аналогами твистованных полей в конформной теории поля с центральным зарядом $c=1$ [11].

Вакуумное ожидание поля беспорядка $\mu(r)$ связано простым соотношением с вакуумным ожиданием экспоненциального поля $\exp (i \nu \phi)$ при $\nu=1 / 2$ в модели синус-Гордон в точке свободных фермионов. Эта модель описывается евклидовым действием

$$
S=\frac{1}{4 \pi} \int d x d \tau\left(\partial_{k} \phi \partial_{k} \phi+\mu \cos (\hat{\beta} \phi)\right)
$$

Точка свободных фермионов соответствует $\hat{\beta}=1$.

Экспоненциальное поле $\exp (i \nu \phi)$ в этой точке определяется следующими коммутационными соотношениями с дираковским полем [12]:

$$
\begin{aligned}
& e^{i \nu \phi(\tau, x)} \psi(\tau, y)=\psi(\tau, y) e^{i \nu \phi(\tau, x)}, \quad y<x, \\
& e^{i \nu \phi(\tau, x)} \psi(\tau, y)=e^{2 \pi i \nu} \psi(\tau, y) e^{i \nu \phi(\tau, x)}, y>x \text {. }
\end{aligned}
$$

Как было показано в работах $[9,10,13]$, в точке свободных фермионов корреляционная функция полей беспорядка $\mu(r)$ удовлетворяет соотношению

$$
\langle\mu(0) \mu(r)\rangle=\left\langle\cos \frac{1}{2} \phi(0) \cos \frac{1}{2} \phi(r)\right\rangle .
$$

Отсюда находим

$$
\langle\mu(0) \mu(r)\rangle=\frac{1}{2}\left\langle e^{i \frac{1}{2} \phi(0)} e^{i \frac{1}{2} \phi(r)}\right\rangle+\frac{1}{2}\left\langle e^{i \frac{1}{2} \phi(0)} e^{-i \frac{1}{2} \phi(r)}\right\rangle .
$$

Это соотношение позволяет получить связь между вакуумными средними поля беспорядка и экспоненциального поля. Заметим, что конкретные вычисления правой и левой частей (1.4) требуют регуляризации и приводят к появлению соответствуюших констант перенормировки волновой функции. Чтобы избавиться от них при вычислении вакуумных средних, удобно нормировать корреляционные функции на поведение при $r \rightarrow 0$. В этом пределе из (1.4) получаем

$$
\langle\mu(0) \mu(r)\rangle=\frac{1}{2}\left\langle e^{i \frac{1}{2} \phi(0)} e^{-i \frac{1}{2} \phi(r)}\right\rangle
$$

где мы воспользовались следуюшей асимптотикой корреляционной функции экспоненциальных полей при $r \rightarrow 0$ [14]:

$$
\left\langle e^{i \nu \phi(0)} e^{i \nu^{\prime} \phi(r)}\right\rangle=\left\langle e^{i\left(\nu+\nu^{\prime}\right) \phi}\right\rangle r^{2 \nu \nu^{\prime}}
$$


Тогда для отношения равенств (1.4) (в пределе $r \rightarrow \infty)$ и (1.5) получаем

$$
\frac{\langle\mu\rangle^{2}}{\left.\langle\mu(0) \mu(r)\rangle\right|_{r \rightarrow 0}}=\frac{2\left\langle e^{i \frac{1}{2} \phi}\right\rangle^{2}}{\left.\left\langle e^{i \frac{1}{2} \phi(0)} e^{-i \frac{1}{2} \phi(r)}\right\rangle\right|_{r \rightarrow 0}} .
$$

Как будет показано ниже, можно выбрать такую нормировку полей беспорядка, чтобы асимптотики корреляционных функций в знаменателях правой и левой частей (1.7) совпадали. В этом случае из (1.7) получаем $\langle\mu\rangle=\sqrt{2}\left\langle\exp \left(i \frac{1}{2} \phi\right)\right\rangle$. В работе [14] было предложено следуюшее выражение для вакуумного ожидания экспоненциального поля:

$$
\left\langle e^{i \nu \phi}\right\rangle=\left(\frac{m}{2}\right)^{\nu^{2}} \exp \left[\int_{0}^{\infty} \frac{d t}{t}\left(\frac{\operatorname{sh}^{2} \frac{\nu t}{2}}{\operatorname{sh}^{2} \frac{t}{2}}-\nu^{2} e^{-t}\right)\right] .
$$

Справедливость этого выражения легко доказывается при помощи результатов вычислений асимптотического поведения корреляционной функции экспоненциальных полей в пределе $r \rightarrow 0$, впервые полученных в [15]. В этой работе исследовалась асимптотика корреляционной функции, которая была вычислена в [12]. Заметим, что здесь для экспоненциальных полей использовалось определение (1.2). Используя результат наших вычислений (см. (4.18) ниже) и выражение (1.8), нетрудно получить соотношение меж ду вакуумными ожиданиями твистованного и экспоненциального полей для $|\nu|<1$ :

$$
\left\langle\mu_{\nu}\right\rangle=4^{\nu^{2}}\langle\exp (i \nu \phi)\rangle
$$

Подчеркнем, что это соотношение справедливо для нормировки, при которой двухточечные корреляционные функции твистованных и экспоненциальных полей имеют одинаковую асимптотику при $r \rightarrow 0$ :

$$
\left\langle\mu_{\nu}(0) \mu_{\nu}(r)\right\rangle \underset{r \rightarrow 0}{=}\left\langle e^{i \nu \phi(0)} e^{-i \nu \phi(r)}\right\rangle \underset{r \rightarrow 0}{=} \frac{1}{r^{2 \nu^{2}}}
$$

В разделе 2 с использованием определения твистованных полей при помощи твистованных граничных условий для дираковских фермионов выводится детерминантное представление коррелящионной функции этих полей через матрищу нетеплицева типа. В разделе 3 рассматривается асимптотика корреляционной функции при $r \rightarrow \infty$ и показывается, что для этого случая можно получить новое детерминантное представление через матрицу теплищева типа. В разделе 4 с помощью полученного представления вычисляется асимптотика корреляционной функции твистованных полей. В приложении приводится вывод некоторых соотношений, которые используются в основном тексте.

\section{2. ПРЕДСТАВЛЕНИЕ КОРРЕЛЯЦИОННОЙ ФУНКЦИИ ТВИСТОВАННЫХ ПОЛЕЙ В ВИДЕ ФУНКЦИОНАЛЬНОГО ИНТЕГРАЛА}

Известно [16, 17], что корреляционная функция полей беспорядка $\left\langle\mu^{\mathrm{I}}(0) \mu^{\mathrm{I}}(r)\right\rangle$ в двумерной модели Изинга может быть представлена в виде функционального интеграла 
теории свободных майорановских фермионов с антипериодическими граничными условиями, за исключением линии корреляции $[0, r]$, где эти фермионы имеют периодические граничные условия.

По аналогии определим корреляционную функцию полей $\mu_{\nu}(r)$ через отношение следующих функциональных интегралов в решеточной теории Дирака:

$$
\left\langle\mu_{\nu}(0) \mu_{\nu}(r)\right\rangle=\frac{\int d[\psi \bar{\psi}] e^{S_{r}[\psi]}}{\int d[\psi \bar{\psi}] e^{S[\psi]}}
$$

с действием

$$
S_{r}[\psi]=(\bar{\psi} \widehat{D} \psi)=\sum_{\rho, \rho^{\prime}} \bar{\psi}(\rho) D_{\rho, \rho^{\prime}} \psi\left(\rho^{\prime}\right),
$$

где $\psi(\rho)$ - комплексное двухкомпонентное грассманово поле, заданное на узлах $\rho=(x, y)$ квадратной решетки размером $n_{x} \times n_{y}, \widehat{D}-$ решеточный оператор Дирака, имеюший вид

$$
\widehat{D}=\left(\begin{array}{cc}
u & v \\
-v^{T} & u^{T}
\end{array}\right)=\left(\begin{array}{cc}
1-t \nabla_{x} & 1-t \nabla_{y} \\
-\left(1-t \nabla_{-y}\right) & 1-t \nabla_{-x}
\end{array}\right) .
$$

Ниже мы покажем, что параметр $t$ связан с массой $m$ в уравнении Дирака следующим образом $\left(a-\right.$ постоянная решетки): $m a=\sqrt{2}\left(t^{-1 / 2}-t^{1 / 2}\right)$. Через $\nabla_{x}, \nabla_{y}$ обозначены операторы сдвига на один шаг решетки соответственно вдоль оси $X$ и $Y$ :

$$
\nabla_{x} \psi(\rho)=\psi(\rho+\hat{x}), \quad \nabla_{y} \psi(\rho)=\psi(\rho+\hat{y}),
$$

где $\hat{x}, \hat{y}$ - соответствующие орты. Индекс $r$ обозначает, что в действии $S_{r}[\psi]$ оператор $\nabla_{y}$ имеет твистованные граничные условия вдоль линии корреляции $[0, r-1](r-1=$ $(r-1,0))$ :

$$
\nabla_{y} \psi\left(x, n_{y}\right)=-e^{2 \pi i \nu} \psi(x, 0), \quad x=0,1, \ldots, r-1 .
$$

В знаменателе формулы (2.1) действие $S[\psi]$ по форме совпадает с $S_{r}[\psi]$, но оператор $\nabla_{y}$ удовлетворяет антипериодическим граничным условиям вдоль всей границы.

Нетрудно проверить, что для оператора $\widehat{D}$ выполняется соотношение $\widehat{D}^{-1}=\widehat{D}^{T} \cdot \widehat{K}$, где $\widehat{K}$ - решеточный аналог оператора Клейна-Гордона

$$
\widehat{K}=\operatorname{det} \widehat{D}=u u^{T}+v v^{T}=2(1-t)^{2}-t\left[\left(\nabla_{x}-2+\nabla_{-x}\right)+\left(\nabla_{y}-2+\nabla_{-y}\right)\right] .
$$

В “наивном" непрерывном пределе (постоянная решетки $a \rightarrow 0) \widehat{K}=a^{2} t\left(m^{2}-\partial_{i} \partial_{i}\right)$, где $\partial_{i}=\left(\nabla_{i}-1\right) / a$ и $m a / \sqrt{2}=t^{-1 / 2}-t^{1 / 2}$. Отсюда для критической точки модели имеем $t_{\mathrm{c}}=1$, и скейлинговая область определяется соотношением $\left|t_{\mathrm{c}}-t\right|=\mathrm{ma} / \sqrt{2}$. В импульсном представлении функция

$$
K(p)=2(1-t)^{2}+4 t\left(\sin ^{2} \frac{p_{x}}{2}+\sin ^{2} \frac{p_{y}}{2}\right),
$$

в отличие от обычного решеточного оператора Дирака, имеет единственный минимум при $p_{x}=p_{y}=0$ в зоне Бриллюэна, и проблем с решеточным дублированием фермионных степеней свободы не возникает. 
Для того чтобы выполнить интегрирование по фермионным полям в числителе (2.1), удобно перейти в действии $S_{r}[\psi]$ к оператору $\nabla_{y}$ с антипериодическими граничными условиями вдоль всей границы, явно указав в $S_{r}[\psi]$ член, содержаший дефект на решетке, обусловленный твистованными граничными условиями,

$$
S_{r}[\psi]=S[\psi]+\delta S_{r}[\psi]
$$

где

$$
\delta S_{r}[\psi]=t \sum_{x=0}^{r-1}\left[-\xi \bar{\psi}^{1}\left(x, n_{y}\right) \psi^{2}(x, 0)+\xi^{*} \bar{\psi}^{2}(x, 0) \psi^{1}\left(x, n_{y}\right)\right]=\left(\bar{\psi} \widehat{P}^{T} \widehat{\Xi} \widehat{P} \psi\right)
$$

Здесь проекционный оператор $\widehat{P}$ выделяет узлы решетки на линии корреляции $[0, r-1]$

$$
\widehat{P}_{l, x}=\delta_{l, x} \delta_{n_{y}, y}\left(\begin{array}{cc}
1 & 0 \\
0 & \nabla_{y}
\end{array}\right), l=0,1, \ldots, r-1
$$

фазовая матрица

$$
\widehat{\Xi}=t\left(\begin{array}{cc}
0 & -\xi \hat{I}^{(r)} \\
\xi^{*} \hat{I}^{(r)} & 0
\end{array}\right)
$$

где $\xi=1-e^{2 \pi i \nu}$ и $\hat{I}^{(r)}-$ единичная матрища размера $r \times r$. Тогда

$$
\left\langle\mu_{\nu}(0) \mu_{\nu}(r)\right\rangle=\left\langle e^{\delta S_{r}[\psi]}\right\rangle=\frac{\int d[\psi \bar{\psi}] e^{S[\psi]+\delta S_{r}[\psi]}}{\int d[\psi \bar{\psi}] e^{S[\psi]}} .
$$

Для вычисления функциональных интегралов в (2.4) удобно представить экспоненту $e^{\delta S_{r}[\psi]}$ через интеграл по вспомогательному полю

$$
e^{\delta S_{r}[\psi]}=|\widehat{\Xi}| \int d[\chi \bar{\chi}] \exp \left[\left(\bar{\chi} \widehat{\Xi}^{-1} \chi\right)+(\bar{\chi} \widehat{P} \psi)+\left(\bar{\psi} \widehat{P}^{T} \chi\right)\right]
$$

Используя (2.5) и выполняя интегрирование в (2.4) по полю $\psi(\rho)$, находим

$$
\begin{aligned}
\left\langle\mu_{\nu}(0) \mu_{\nu}(r)\right\rangle & =|\widehat{\Xi}| \int d[\chi \bar{\chi}] \exp \left[\left(\bar{\chi} \widehat{\Xi}^{-1} \chi\right)+\left(\bar{\chi} \widehat{P} \widehat{D}^{-1} \widehat{P}^{T} \chi\right)\right]= \\
& =|\widehat{\Xi}|\left|\widehat{\Xi}^{-1}+\widehat{P} \widehat{D}^{-1} \widehat{P}^{T}\right|=|\widehat{G}|,
\end{aligned}
$$

где $|\widehat{G}|$ обозначает детерминант блочной $(2 \times 2)$-матрицы с блоками размера $r \times r$. Матрица $\widehat{G}$ имеет вид

$$
\widehat{G}=\left(\begin{array}{cc}
g_{x, x^{\prime}}^{11} \sin \pi \nu & -g_{x, x^{\prime}}^{12} \sin \pi \nu+i I_{x, x^{\prime}}^{(r)} \cos \pi \nu \\
g_{x, x^{\prime}}^{12} \sin \pi \nu+i I_{x, x^{\prime}}^{(r)} \cos \pi \nu & \left(g_{x, x^{\prime}}^{11}\right)^{T} \sin \pi \nu
\end{array}\right)
$$

где

$$
\begin{aligned}
& g_{x, x^{\prime}}^{11}=\frac{1}{(2 \pi)^{2}} \int_{-\pi}^{\pi} \frac{d^{2} p}{K(p)} e^{i p_{x}\left(x-x^{\prime}\right)} 2 t u^{*}\left(p_{x}\right), \quad u\left(p_{x}\right)=1-t e^{i p_{x}}, \\
& g_{x, x^{\prime}}^{12}=\frac{1}{(2 \pi)^{2}} \int_{-\pi}^{\pi} \frac{d^{2} p}{K(p)} e^{i p_{x}\left(x-x^{\prime}\right)} 2 t\left(u^{*}\left(p_{x}\right)+u\left(p_{x}\right)\right) .
\end{aligned}
$$


Покажем, что выражение для корреляционной функции (2.6) при $\nu=1 / 2$ совпадает с квадратом корреляционной функции полей беспорядка в модели Изинга. Для этого сделаем преобразование подобия матрицы $\widehat{G}$ с помошью унитарной матрищы $\widehat{Q}$

$$
\widehat{Q}=e^{i \gamma_{5} \frac{\theta}{2}}, \quad \gamma_{5}=\left(\begin{array}{cc}
0 & -i \\
i & 0
\end{array}\right), \quad \operatorname{ctg} \theta=t
$$

В результате получаем

$$
G(r)=\left\langle\mu_{\nu}(0) \mu_{\nu}(r)\right\rangle \equiv\left|\widehat{Q} \cdot \widehat{G} \cdot \widehat{Q}^{-1}\right|=\left|\cos ^{2} \pi \nu+\sin ^{2} \pi \nu \widehat{V} \cdot \widehat{V}^{T}\right|
$$

где

$$
\begin{gathered}
V_{x, x^{\prime}}=\frac{1}{2 \pi i} \oint_{|z|=1} \frac{d z}{z} z^{-\left(x-x^{\prime}\right)} V(z), \quad V(z)=\sqrt{\frac{(1-\alpha z)\left(1-\beta z^{-1}\right)}{\left(1-\alpha z^{-1}\right)(1-\beta z)}} \\
\alpha=t \frac{\sqrt{1+t^{2}}+t}{\sqrt{1+t^{2}}+1}, \quad \beta=t \frac{\sqrt{1+t^{2}}-t}{\sqrt{1+t^{2}}+1}
\end{gathered}
$$

Здесь матрища $V_{x, x^{\prime}}$ в точности совпадает с классическим выражением для теплицевой матрицы [18], через определитель которой выражается парная корреляционная функция поля беспорядка $\left\langle\mu^{\mathrm{I}}(0) \mu^{\mathrm{I}}(r)\right\rangle$ в модели Изинга в парамагнитной области. Для некоторой экономии в дальнейшем мы не будем выписывать множитель $(2 \pi i)^{-1}$ в контурных интегралах, подразумевая, что он включен должным образом в меру интегрирования. Очевидно, что при $\nu=1 / 2$ из (2.9) следует (1.1).

Отметим, что преобразование $\widehat{Q}$ связано с конкретным выбором решеточного варианта оператора Дирака (2.3). В “наивном” непрерьвном пределе действие (2.2) принимает вид

$$
S[\psi]=\int d^{2} \rho \bar{\psi}(\rho)\left(m e^{i \gamma_{5} \frac{\pi}{4}}-\gamma_{i} \partial_{i}\right) \psi(\rho)
$$

где

$$
\gamma_{x}=\left(\begin{array}{cc}
1 & 0 \\
0 & -1
\end{array}\right), \quad \gamma_{y}=\left(\begin{array}{cc}
0 & -i \\
i & 0
\end{array}\right)
$$

В критической точке $t=t_{\mathrm{c}}=1$ угол $\theta$ в (2.8) равен $\pi / 4$ и преобразование $\widehat{Q}$ сводится к $\gamma_{5}$-повороту грассмановых полей: $\psi^{\prime}=\exp \left(i \gamma_{5} \pi / 8\right) \psi$, после чего дираковское действие принимает обычный вид

$$
S[\psi]=\int d^{2} \rho \bar{\psi}(\rho)\left(m-\gamma_{i} \partial_{i}\right) \psi(\rho)
$$




\section{3. ПРЕДСТАВЛЕНИЕ КОРРЕЛЯЦИОННОЙ ФУНКЦИИ В ВИДЕ ТЕПЛИЦЕВА ДЕТЕРМИНАНТА}

В этом разделе мы покажем, что при $r \rightarrow \infty$ и в скейлинговом пределе $(m a \rightarrow 0)$ вычисление корреляционной функции (2.9) можно свести к вычислению детерминанта матрицы теплицева типа:

$$
G(r)=\left|\cos ^{2} \pi \nu+\sin ^{2} \pi \nu \widehat{V} \cdot \widehat{V}^{T}\right| \underset{r \rightarrow \infty}{=}\left|V^{(\nu)} \cdot V^{(\nu)^{T}}\right|=\left|V^{(\nu)}\right|^{2},
$$

где

$$
V_{x, x^{\prime}}^{(\nu)}=\oint_{|z|=1} \frac{d z}{z} z^{-\left(x-x^{\prime}\right)} V^{(\nu)}(z), \quad V^{(\nu)}(z)=\left[\frac{(1-\alpha z)\left(1-\beta z^{-1}\right)}{\left(1-\alpha z^{-1}\right)(1-\beta z)}\right]^{\nu} .
$$

При $\nu=1 / 2$ ядро $V^{(\nu)}(z)$ совпадает с ядром $V(z)(2.10)$.

В приложении показано, что корреляционную функцию (2.9) можно представить в следующем виде:

$$
G(r) \underset{r \rightarrow \infty}{=}\left|1-\sin ^{2} \pi \nu \hat{A}\right|^{2},
$$

где

$$
A_{x, x^{\prime}}=\oint_{\left|z_{1} z_{2}\right|<1} \frac{d z_{1} d z_{2}\left(z_{1}\right)^{x}\left(z_{2}\right)^{x^{\prime}}}{\left(1-z_{1} z_{2}\right)} V^{-1}\left(z_{1}\right) V^{-1}\left(z_{2}\right) .
$$

Здесь контуры интегрирования проходят между разрезами, изображенными на рисунке.

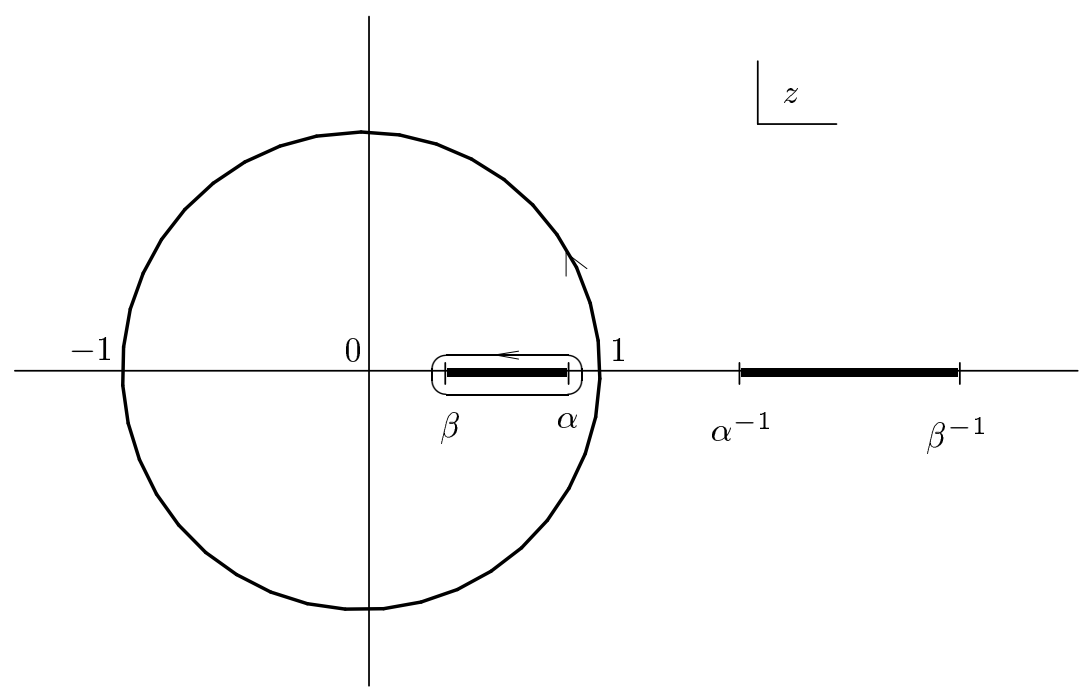


Используя (3.3), получаем

$$
\frac{1}{2} \ln G(r)=\operatorname{Sp} \ln \left(1-\sin ^{2} \pi \nu \hat{A}\right)=-\sum_{k=1}^{\infty} \frac{a_{k}}{k}\left(\frac{\sin \pi \nu}{\pi}\right)^{2 k}
$$

где

$$
a_{k}=\pi^{2 k} \operatorname{Sp} \hat{A}^{k}=\pi^{2 k} \oint \prod_{l=1}^{2 k} d z_{l} \frac{\prod_{l=1}^{k}\left[1-\left(z_{l} z_{l+1}\right)^{r}\right]}{\prod_{l=1}^{2 k}\left[\left(1-z_{l} z_{l+1}\right) V\left(z_{l}\right)\right]}, \quad z_{2 l+1}=z_{1} .
$$

При $r \rightarrow \infty$ слагаемые в правой части $(3.5)$, содержащие произведения $\left(z_{l} z_{l+1}\right)^{r}$, экспоненциально малы, так что

$$
\begin{aligned}
a_{k} & =\pi^{2 k} \oint \frac{\prod_{l=1}^{2 k} d z_{l}}{\prod_{l=1}^{2 k}\left[\left(1-z_{l} z_{l+1}\right) V\left(z_{l}\right)\right]}= \\
& =\int_{\beta}^{\alpha} \frac{\prod_{l=1}^{2 k} d z_{l}}{\prod_{l=1}^{2 k}\left(1-z_{l} z_{l+1}\right)} \prod_{l=1}^{2 k}\left[\frac{\left(\alpha-z_{l}\right)\left(1-\beta z_{l}\right)}{\left(1-\alpha z_{l}\right)\left(z_{l}-\beta\right)}\right]^{\frac{1}{2}}
\end{aligned}
$$

В пределе $m a=0$, т.е. $\alpha=1$, интеграл в (3.6) расходится логарифмически на верхнем пределе (в окрестности “правого верхнего угла" $2 k$-мерного гиперкуба с координатами $\left.z_{l}=1\right)$. Выделим и оценим эту расходимость:

$$
a_{k} \simeq \int_{\alpha-\varepsilon}^{\alpha} \prod_{l=1}^{2 k} \frac{d z_{l}}{1-z_{l} z_{l+1}} \prod_{l=1}^{2 k}\left(\frac{\alpha-z_{l}}{1-\alpha z_{l}}\right)^{\frac{1}{2}} \simeq \int_{1}^{\varepsilon / m a} \prod_{l=1}^{2 k} \frac{d z_{l}}{z_{l}+z_{l+1}}
$$

Для вывода этой оценки мы сделали ряд последовательных замен переменных: $z_{l}=\alpha-$ $z_{l}^{\prime}, z_{l}^{\prime}=(m a) z_{l}^{\prime \prime}, z_{l}^{\prime \prime}=z_{l}+1$. Явно выделив в последнем интеграле логарифмическую сингулярность, получаем равенство

$$
a_{k}=\int_{1}^{\varepsilon / m a} \prod_{l=1}^{2 k} \frac{d z_{l}}{z_{l}+z_{l+1}}=-k b_{k} \ln (m a)+\text { const } .
$$

Коэффициенты $b_{k}$ можно выразить через многократные интегралы, продифференцировав по та правую и левую части (3.8),

$$
b_{k}=2 \int_{0}^{1} \prod_{l=1}^{2 k-1} d z_{l}\left[\left(1+z_{1}\right)\left(1+z_{2 k-1}\right) \prod_{l=1}^{2 k-2}\left(z_{l}+z_{l+1}\right)\right]^{-1} .
$$

Используя формулы (3.8) и (3.4), получаем следующее асимптотическое выражение для корреляционной функции при $r \rightarrow \infty, m a \rightarrow 0$ :

$$
\frac{1}{2} \ln G(\infty)=\ln (m a) \sum_{k=1}^{\infty} b_{k}\left(\frac{\sin \pi \nu}{\pi}\right)^{2 k}+\text { const }
$$


Интегралы для первых трех коэффициентов в (3.9) можно свести к табличным:

$$
b_{1}=1, \quad b_{2}=2 \zeta(2), \quad b_{3}=4 \zeta^{2}(2)+6 \zeta(4),
$$

где $\zeta(k)-\zeta$-функция Римана

$$
\zeta(k+1)=\frac{(-1)^{k}}{k !} \int_{0}^{1} \frac{d x \ln ^{k} x}{1-x} .
$$

Раскладывая функции $(\sin \pi \nu / \pi)^{2 k}$, входящие в ряд (3.10), по степеням $\nu$ и используя значения (3.11) для коэффициентов $b_{k}$, нетрудно убедиться в том, что

$$
\begin{aligned}
& b_{1} \frac{\sin ^{2} \pi \nu}{\pi^{2}}=\nu^{2}+\mathrm{O}\left(\nu^{4}\right), \\
& b_{1} \frac{\sin ^{2} \pi \nu}{\pi^{2}}+b_{2} \frac{\sin ^{4} \pi \nu}{\pi^{4}}=\nu^{2}+\mathrm{O}\left(\nu^{6}\right), \\
& b_{1} \frac{\sin ^{2} \pi \nu}{\pi^{2}}+b_{2} \frac{\sin ^{4} \pi \nu}{\pi^{4}}+b_{3} \frac{\sin ^{6} \pi \nu}{\pi^{6}}=\nu^{2}+\mathrm{O}\left(\nu^{8}\right),
\end{aligned}
$$

т.е. каждьй следующий член в ряду (3.11) уничтожает все более высокие степени $\nu^{2 k}$. Хотя прямое вычисление интегралов (3.9) кратности выше пятой вряд ли выполнимо, можно предположить, что и в более высоких порядках соотношения типа (3.12) имеют место, т.е.

$$
\sum_{k=1}^{\infty} b_{k}\left(\frac{\sin \pi \nu}{\pi}\right)^{2 k}=\nu^{2} .
$$

Это означает, что производящей функцией для коэффициентов $b_{k}$ является функция

$$
\frac{1}{\pi^{2}} \arcsin ^{2}(\pi \sqrt{z})=\sum_{k=1}^{\infty} b_{k} z^{k} .
$$

Если это действительно так, то для определителя (3.1) имеет место следующее выражение:

$$
G(\infty) \underset{(m a) \rightarrow 0}{=} \operatorname{const} \cdot(m a)^{2 \nu^{2}} .
$$

Заметим, что формула (3.14) позволяет находить значения интегралов (3.9) произвольной кратности.

Докажем справедливость соотношений (3.14) и (3.15). Ключевым для доказательства является следуюшее обстоятельство, вытекаюшее из цепочки выкладок (3.7): при асимптотической $(m a \rightarrow 0)$ оценке интегралов в (3.6) оказывается несушественным показатель степени в выражении, задаюшем ядро матрицы $V_{x, x^{\prime}}$, что позволяет ввести новую матрицу $V_{x, x^{\prime}}^{(\nu)}$ с ядром (3.2). Для этого определим корреляционную функцию

$$
G^{(\nu)}(r)=\left|\widehat{V}^{(\nu)} \cdot \widehat{V}^{(\nu) T}\right|
$$


где матрица $\widehat{V}^{(\nu)}$ определена в (3.2). Используя результаты приложения, функцию (3.16) можно представить следуюшим образом:

$$
G^{(\nu)}(r) \underset{r \rightarrow \infty}{=}\left|1-\hat{A}^{(\nu)}\right|^{2}
$$

где матрица $\hat{A}^{(\nu)}$ имеет вид

$$
A_{x, x^{\prime}}^{(\nu)}=\oint \frac{d z_{1} d z_{2}\left(z_{1}\right)^{x}\left(z_{2}\right)^{x^{\prime}}}{\left(1-z_{1} z_{2}\right)} \frac{1}{V^{(\nu)}\left(z_{1}\right) V^{(\nu)}\left(z_{2}\right)} .
$$

След от $k$-й степени матрицы $\hat{A}^{(\nu)}$ выражается через $2 k$-кратньй интеграл

$$
\begin{aligned}
\operatorname{Sp}\left[\left(\hat{A}^{(\nu)}\right)^{k}\right]= & \left(\frac{\sin \pi \nu}{\pi}\right)^{2 k} \int_{\beta}^{\alpha} \prod_{l=1}^{2 k} \frac{d z_{l}}{\left(1-z_{l} z_{l+1}\right)} \times \\
& \times \prod_{l=1}^{2 k}\left[\frac{\left(\alpha-z_{l}\right)\left(1-\beta z_{l}\right)}{\left(1-\alpha z_{l}\right)\left(z_{l}-\beta\right)}\right]^{\nu} \prod_{l=1}^{k}\left[1-\left(z_{l} z_{l+1}\right)^{r}\right] .
\end{aligned}
$$

Здесь, в отличие от формулы (3.4), множитель $(\sin \pi \nu)^{2 k}$ возникает при стягивании контура интегрирования в (3.18) на разрез $[\beta, \alpha]$ за счет показателя $\nu$, задаюшего тип точек ветвления в ядре (3.2). С учетом (3.19) для $G^{(\nu)}(r)$ при $r \rightarrow \infty$ получаем выражение, аналогичное (3.4),

$$
\frac{1}{2} \ln G^{(\nu)}(r)=\operatorname{Sp} \ln \left(1-\hat{A}^{(\nu)}\right)=-\sum_{k=1}^{\infty} \frac{a_{k}^{(\nu)}}{k}\left(\frac{\sin \pi \nu}{\pi}\right)^{2 k},
$$

где

$$
a_{k}^{(\nu)}=\int_{\beta}^{\alpha} \prod_{l=1}^{2 k} \frac{d z_{l}}{\left(1-z_{l} z_{l+1}\right)} \prod_{l=1}^{2 k}\left[\frac{\left(\alpha-z_{l}\right)\left(1-\beta z_{l}\right)}{\left(1-\alpha z_{l}\right)\left(z_{l}-\beta\right)}\right]^{\nu} \prod_{l=1}^{k}\left[1-\left(z_{l} z_{l+1}\right)^{r}\right] .
$$

При $r \rightarrow \infty$ слагаемые в (3.21), содержашие степени $\left(z_{l} z_{l+1}\right)^{r}$, исчезают. Оставшиеся интегралы в (3.21) расходятся на верхнем пределе при $\alpha \rightarrow 1$, однако разность между $(3.6)$ и $(3.21)$

$$
\begin{aligned}
\Delta a_{k}^{(\nu)} \equiv & a_{k}-a_{k}^{(\nu)}=\int_{\beta}^{\alpha} \prod_{l=1}^{2 k} \frac{d z_{l}}{\left(1-z_{l} z_{l+1}\right)} \times \\
& \times\left\{\prod_{l=1}^{2 k}\left[\frac{\left(\alpha-z_{l}\right)\left(1-\beta z_{l}\right)}{\left(1-\alpha z_{l}\right)\left(z_{l}-\beta\right)}\right]^{\frac{1}{2}}-\prod_{l=1}^{2 k}\left[\frac{\left(\alpha-z_{l}\right)\left(1-\beta z_{l}\right)}{\left(1-\alpha z_{l}\right)\left(z_{l}-\beta\right)}\right]^{\nu}\right\}
\end{aligned}
$$

является конечной величиной при $\alpha=1$,

$$
\Delta a_{k}^{(\nu)}=\sqrt{2}\left(\frac{1}{2}-\nu\right) \int_{1-\varepsilon}^{1} \prod_{l=1}^{2 k} \frac{d z_{l}}{\left(1-z_{l} z_{l+1}\right)} \sum_{l=1}^{2 k}\left(1-z_{l}\right)+\text { const }=\text { const } .
$$


Отсюда следует, что отношение детерминантов (2.9) и (3.16) также является конечным и не равным нулю:

$$
\gamma \equiv \frac{G(\infty)}{G^{(\nu)}(\infty)}=\frac{\left|\cos ^{2} \pi \nu+\sin ^{2} \pi \nu \widehat{V} \cdot \widehat{V}^{T}\right|}{\left|\widehat{V}^{(\nu)} \cdot \widehat{V}^{(\nu) T}\right|} \underset{m a \rightarrow 0}{=} \text { const }
$$

Поэтому сингулярный по та фактор в $G(\infty)$ совпадает с таковым в определителе (3.16). Поскольку матрица $\widehat{V}^{(\nu)}$ является теплицевой, а теория теплицевых детерминантов хорошо развита, то задача по вычислению (2.9) в пределе $r \rightarrow \infty, m a \rightarrow 0$ может быть решена.

Отношение детерминантов (3.23) рассматривалось нами в пределе $r=\infty, m a \rightarrow 0$. В работе [14] коррелятор экспоненциальных полей был нормирован на корреляционную функцию этих полей в теории безмассовых дираковских фермионов. Поэтому для сравнения наших результатов с [14] необходимо исследовать отношение (3.23) в пределе $m a=0, r \rightarrow \infty$. Точнее говоря, надо убедиться, что величина $\gamma$ не зависит от последовательности предельных переходов.

Можно показать, что при $m a=0$ разность коэффициентов (3.5) и (3.21) отличается от (3.22) на некоторую добавку, убываюшую с ростом $r$,

$$
a_{k}-a_{k}^{(\nu)}=\Delta a_{k}+\frac{\phi_{k}(r)}{r^{2}}
$$

Асимптотическая оценка для $\phi_{k}(r)$ имеет вид

$$
\phi_{k}(r) \simeq \frac{\ln ^{k} r}{k !}
$$

В результате при $m a=0, r \rightarrow \infty$ получаем

$$
\frac{G(r)}{G^{(\nu)}(r)}=\gamma \exp \left[\frac{1}{r^{2}} \sum_{k=1}^{\infty} \phi_{k}(r)\right]
$$

Для лидирующих по $r$ вкладов $(3.24)$ в $\phi_{k}(r)$ суммирование в экспоненте правой части (3.25) выполняется в явном виде, что приводит к оценке

$$
\frac{G(r)}{G^{(\nu)}(r)}=\gamma+\mathrm{O}\left(\frac{1}{r}\right)
$$




\section{4. АСИМПТОТИКА КОРРЕЛЯЦИОННОЙ ФУНКЦИИ}

Здесь мы вычисляем асимптотику корреляционной функции $G(r)$, используя для нее представление (3.1) через детерминант матрицы $V_{x, x^{\prime}}^{(\nu)}$. Поскольку эта матрица относится к теплицеву типу, для исследования асимптотического поведения ее детерминанта можно применить технику работы [18], использовавшуюся при исследовании асимптотического поведения корреляционной функции в модели Изинга $(\nu=1 / 2)$. Для случая произвольного $0<\nu<1$ эта техника требует некоторого обобщения. Из-за громоздкости выкладок приведем только окончательный результат:

$$
\ln \left|\widehat{V}^{(\nu)}\right| \underset{r \rightarrow \infty}{=} r \oint \frac{d z}{z} \ln V^{(\nu)}(z)-\oint d z \ln P(z) \frac{\partial}{\partial z} \ln Q\left(z^{-1}\right) .
$$

Подставляя сюда явные выражения для функций $P(z)$ и $Q(z)$ :

$$
V^{(\nu)}(z)=P(z) Q\left(z^{-1}\right), \quad P(z)=\left(\frac{1-\alpha z}{1-\beta z}\right)^{\nu}, \quad Q(z)=\left(\frac{1-\beta z}{1-\alpha z}\right)^{\nu}=\frac{1}{P(z)},
$$

получаем

$$
\begin{aligned}
\oint \frac{d z}{z} \ln V^{(\nu)}=\nu\left(\oint \frac{d z}{z} \ln P(z)\right. & \left.-\oint \frac{d z}{z} \ln P\left(z^{-1}\right)\right)=\nu(P(0)-P(0))=0 \\
-\oint d z \ln P(z) \frac{\partial}{\partial z} \ln Q\left(z^{-1}\right) & =\nu^{2} \oint d z \ln P(z)\left(\frac{1}{z-\alpha}-\frac{1}{z-\beta}\right)= \\
& =\nu^{2}(\ln P(\alpha)-\ln P(\beta))
\end{aligned}
$$

В результате имеем

$$
\left|\widehat{V}^{(\nu)}\right| \underset{r \rightarrow \infty}{=}\left(\frac{P(\alpha)}{P(\beta)}\right)^{\nu^{2}}=\left[\frac{\left(1-\alpha^{2}\right)\left(1-\beta^{2}\right)}{(1-\alpha \beta)^{2}}\right]^{\nu^{2}} .
$$

Заметим, что данное выражение выведено при конечных значениях $m a$ и $r$. Вывод (4.1) справедлив и в скейлинговом режиме: $m a \rightarrow 0, r \rightarrow \infty, r(m a)=$ const. Однако при $m a=0, r=$ const интегралы в правой части (4.1) являются расходяшимися и вычисление $\left|\widehat{V}^{(\nu)}\right|$ при $m a=0$ и $r \rightarrow \infty$ требует специального рассмотрения.

Прежде чем перейти к этому, упростим немного задачу, заметив, что отношение детерминантов $\left|\widehat{V}^{(\nu)}(\beta)\right| /\left|\widehat{V}^{(\nu)}(0)\right|$ является конечной величиной как в скейлинговом режиме, так и в критической точке, а именно

$$
\frac{\left|\widehat{V}^{(\nu)}(\beta)\right|}{\left|\widehat{V}^{(\nu)}(0)\right|} \underset{r \rightarrow \infty}{=}\left[\frac{1-\beta^{2}}{(1-\alpha \beta)^{2}}\right]^{\nu^{2}} \underset{m a=0}{=} 2^{\nu^{2} / 2}
$$

Здесь явно указана зависимость матрицы $\widehat{V}^{(\nu)}$ от параметра $\beta$ : матрица $\widehat{V}^{(\nu)}(0)$ определяется ядром (3.2) при значении $\beta=0$. 
Соотношение (4.4) нетрудно вывести, используя (4.1). Действительно, для отношения детерминантов в левой части (4.4) получаем

$$
\begin{aligned}
\ln \left(\frac{\left|\widehat{V}^{(\nu)}(\beta)\right|}{\left|\widehat{V}^{(\nu)}(0)\right|}\right)= & -\oint d z \ln P(z) \frac{\partial}{\partial z} \ln Q\left(z^{-1}\right)+ \\
& +\oint d z[\ln P(z)+\nu \ln (1-\beta z)] \frac{\partial}{\partial z}\left[\ln Q\left(z^{-1}\right)-\nu \ln \left(1-\beta z^{-1}\right)\right]= \\
= & -\nu \ln P(\beta)+\nu \ln P(0)-\nu^{2} \ln \left(1-\beta^{2}\right)+ \\
& +\nu \oint d z \ln (1-\beta z) \frac{\partial}{\partial z} \ln Q\left(z^{-1}\right) .
\end{aligned}
$$

Последнее слагаемое в правой части (4.5) проинтегрируем по частям:

$$
\nu \oint d z \ln (1-\beta z) \frac{\partial}{\partial z} \ln Q\left(z^{-1}\right)=\nu \beta \oint \frac{d z}{1-\beta z} \ln Q(z)=\nu \ln Q(\beta) .
$$

В результате найдем, что

$$
\ln \left(\frac{\left|\widehat{V}^{(\nu)}(\beta)\right|}{\left|\widehat{V}^{(\nu)}(0)\right|}\right)=-\nu \ln P(\beta)+\nu \ln Q(\beta)-\nu^{2} \ln \left(1-\beta^{2}\right)=\ln \left[\frac{1-\beta^{2}}{(1-\alpha \beta)^{2}}\right]^{\nu^{2}}
$$

Матричные элементы матрищы

$$
V_{x, x^{\prime}}^{(\nu)}(0)=\left.\oint \frac{d z}{z} z^{-\left(x-x^{\prime}\right)} V^{(\nu)}(z)\right|_{\beta=0}
$$

при $m a=0(\alpha=1)$ вычисляются в явном виде:

$$
V_{x, x^{\prime}}^{(\nu)}(0)=\frac{\sin \pi \nu}{\pi} \frac{1}{\nu-x+x^{\prime}} .
$$

Напомним, что величины $x, x^{\prime}$ пробегают значения $0, \ldots, r-1$. Чтобы упростить обозначения, введем матрицу

$$
\widehat{U}_{r} \equiv V_{x, x^{\prime}}^{(\nu)}(0)
$$

где нижний индекс у матрицы (4.6) явно указывает на ее размерность. Умножив матрицу (4.6) слева на левую треугольную матрицу $\hat{L}_{r}$ и справа на правую треугольную матрицу $\widehat{R}_{r}$, получим соотношение

$$
\hat{L}_{r} \cdot \widehat{U}_{r} \cdot \widehat{R}_{r}=\left(\begin{array}{cc}
\frac{\sin \pi \nu}{\pi} & \hat{0} \\
\hat{0} & -\widehat{U}_{r-1}
\end{array}\right)
$$

где матрицы $\hat{L}_{r}$ и $\widehat{R}_{r}$ имеют следуюший вид:

$$
\hat{L}_{r}=\left(\begin{array}{cccc}
1 & 0 & \ldots & 0 \\
-\frac{1}{1} & \frac{\nu}{1}-1 & \ldots & 0 \\
\vdots & \vdots & \ddots & \vdots \\
-\frac{1}{r-1} & 0 & \ldots & \frac{\nu}{r-1}-1
\end{array}\right), \quad \widehat{R}_{r}=\left(\begin{array}{cccc}
\nu & -\frac{1}{1} & \ldots & -\frac{1}{r-1} \\
0 & \frac{\nu}{1}+1 & \ldots & 0 \\
\vdots & \vdots & \ddots & \vdots \\
0 & 0 & \ldots & \frac{\nu}{r-1}+1
\end{array}\right)
$$


Детерминант произведения этих матриц нетрудно вычислить:

$$
\left|\hat{L}_{r} \cdot \widehat{R}_{r}\right|=\frac{(-1)^{r-1} \sin \pi \nu}{\pi} \frac{\Gamma(r+\nu) \Gamma(r-\nu)}{\Gamma^{2}(r)} .
$$

Это позволяет получить из формул (4.8), (4.10) простое рекуррентное соотношение для определителей

$$
\left|\widehat{U}_{r-1}\right|=\left|\widehat{U}_{r}\right| \frac{\Gamma(r+\nu) \Gamma(r-\nu)}{\Gamma^{2}(r)},
$$

решением которого является

$$
\left|\widehat{U}_{r}\right|=\prod_{k=1}^{r} \frac{\Gamma^{2}(k)}{\Gamma(k+\nu) \Gamma(k-\nu)} .
$$

Воспользовавшись следуюшим представлением для Г-функции:

$$
\ln \Gamma(z)=\int_{0}^{\infty} \frac{d t}{t}\left(\frac{e^{z t}-e^{-t}}{1-e^{-t}}+(z-1) e^{-t}\right),
$$

получим для (4.11) выражение

$$
\ln \left|\widehat{U}_{r}\right|=-\int_{0}^{\infty} \frac{d t}{t}\left(1-e^{-t r}\right) \frac{\operatorname{sh}^{2} \frac{\nu t}{2}}{\operatorname{sh}^{2} \frac{t}{2}}
$$

При $r \rightarrow \infty$ интеграл в правой части (4.12) расходится в нуле. Выделим эту расходимость, переписав (4.12) в виде

$$
\ln \left|\widehat{U}_{r}\right|=-\nu^{2} \ln r-\int_{0}^{\infty} \frac{d t}{t}\left(\frac{\operatorname{sh}^{2} \frac{\nu t}{2}}{\operatorname{sh}^{2} \frac{t}{2}}-\nu^{2} e^{-t}\right)+\int_{0}^{\infty} \frac{d t}{t} e^{-t r}\left(\frac{\operatorname{sh}^{2} \frac{\nu t}{2}}{\operatorname{sh}^{2} \frac{t}{2}}-\nu^{2}\right) .
$$

Поскольку зависяший от $r$ интеграл теперь хорошо определен, получим для асимптотики по $r$ соотношение

$$
\ln \left|\widehat{U}_{r}\right| \underset{r \rightarrow \infty}{=}-\nu^{2} \ln r-\int_{0}^{\infty} \frac{d t}{t}\left(\frac{\operatorname{sh}^{2} \frac{\nu t}{2}}{\operatorname{sh}^{2} \frac{t}{2}}-\nu^{2} e^{-t}\right)+\mathrm{O}\left(\frac{1}{r^{2}}\right) .
$$

С учетом формул (3.1), (4.3), (4.4), (4.7) и (4.13) находим для корреляционной функции $G(r)$ следуюшие асимптотические выражения:

$$
\begin{aligned}
& G(r) \underset{r \rightarrow \infty, \alpha<1}{=}\left[\frac{\left(1-\alpha^{2}\right)\left(1-\beta^{2}\right)}{(1-\alpha \beta)^{2}}\right]^{2 \nu^{2}}=(2 \sqrt{2} m a)^{2 \nu^{2}} \\
& G(r) \underset{r \rightarrow \infty, \alpha=1}{=}\left[\frac{\left(1-\beta^{2}\right)}{r(1-\alpha \beta)^{2}}\right]^{2 \nu^{2}} \exp \left[-2 \int_{0}^{\infty} \frac{d t}{t}\left(\frac{\operatorname{sh}^{2} \frac{\nu t}{2}}{\operatorname{sh}^{2} \frac{t}{2}}-\nu^{2} e^{-t}\right)\right] .
\end{aligned}
$$


Определяя не зависяшую от нормировки и параметра решеточного обрезания величину $h(R m)$ как отношение выражений (4.14) и (4.15) и учитывая (4.4), получаем

$$
h(R m)=\frac{\left.G(r)\right|_{m \neq 0}}{\left.G(r)\right|_{m=0}}=(2 m R)^{2 \nu^{2}} \exp \left[2 \int_{0}^{\infty} \frac{d t}{t}\left(\frac{\operatorname{sh}^{2} \frac{\nu t}{2}}{\operatorname{sh}^{2} \frac{t}{2}}-\nu^{2} e^{-t}\right)\right],
$$

где через $R=r a$ обозначено размерное расстояние.

Для сравнения этой асимптотики с результатами работы [14] в точке свободных фермионов заметим, что авторы [14] использовали следующую нормировку для корреляционной функции в безмассовом случае:

$$
G(R)=R^{-2 \nu^{2}}
$$

Воспользовавшись этой нормировкой, из отношения (4.16) получаем

$$
G(R)_{R \rightarrow \infty, m \neq 0}\left\langle\mu_{\mu}\right\rangle^{2}=(2 m)^{2 \nu^{2}} \exp \left[2 \int_{0}^{\infty} \frac{d t}{t}\left(\frac{\operatorname{sh}^{2} \frac{\nu t}{2}}{\operatorname{sh}^{2} \frac{t}{2}}-\nu^{2} e^{-t}\right)\right] .
$$

\section{5. ЗАКЛЮЧЕНИЕ}

В данной работе получено детерминантное представление для корреляционной функции твистованных полей беспорядка в двумерной решеточной теории Дирака. Вычислив асимптотическое поведение этой корреляционной функции, мы нашли вакуумное ожидание твистованного поля. Полученное выражение отличается от вакуумного ожидания экспоненциального поля на константу, зависящую от индекса твистования $\nu$.

Можно привести следующие аргументы для объяснения этого факта. Известно, что квантовые поля, удовлетворяющие коммутационным соотношениям (1.2), реализуют операторное решение задачи изомонодромной деформации решений уравнения Дирака с нетривиальной монодромией [12]. Корреляционную функцию твистованных полей (2.1) можно интерпретировать как функциональный интеграл в теории Дирака с заданными условиями монодромии для фермионных полей, рассматриваемый в рамках решеточной регуляризации. Тогда такие корреляционные функции могут быть связаны с другим решением задачи изомонодромной деформации уравнения Дирака. Для проверки этого предположения необходимо вычислить детерминант (2.9) и вывести дифференциальное уравнение для корреляционной функции твистованных полей.

Заметим, что с нашей точки зрения в приложении В работы [14] вместо вакуумного ожидания экспоненциального поля вычислялось вакуумное ожидание твистованного поля, однако использование нормировки, согласующейся с вычислениями асимптотики корреляционной функции экспоненциальных полей при $r \rightarrow 0$ [15], привело к правильному результату (1.8) для вакуумного ожидания экспоненциальных полей.

Благодарности. Авторы благодарят Н. А. Славнова, С.З. Пакуляка, С. Лукьянова и М. Лашкевича за полезные обсуждения. Один из авторов (В.Ш.) благодарит А. Ю. Морозова за поддержку и гостеприимство в ИТЭФ, где эта работа была закончена. Работа выполнена при поддержке программы INTAS (грант № 97-1312). 


\section{ПРИЛОЖЕНИЕ}

Покажем, что при $r \rightarrow \infty$ справедливо следуюшее представление для детерминанта:

$$
g(r)=\left|\cos ^{2} \pi \nu^{\prime}+\sin ^{2} \pi \nu^{\prime} \widehat{C} \cdot \widehat{C}^{T}\right| \underset{r \rightarrow \infty}{=}\left|1-\sin ^{2} \pi \nu^{\prime} \hat{A}\right|^{2},
$$

где матрица

$$
C_{x, y}=\oint_{|z|=1} \frac{d z}{z} z^{-(x-y)} C(z), \quad C(z)=\left[\frac{(1-\alpha z)\left(1-\beta z^{-1}\right)}{\left(1-\alpha z^{-1}\right)(1-\beta z)}\right]^{\mu}
$$

для удобства определена с размерами $(r+1) \times(r+1)(x, y=0,1, \ldots, r)$ и $0<\mu<1$. При $\mu=1 / 2$ эта матрица совпадает с матрицей $V_{x, y}$ в детерминантном представлении корреляционной функции (2.9). В соотношении (П.1) матрица $\hat{A}$ задана соотношением

$$
A_{x, y}=\oint_{\left|z_{1} z_{2}\right|<1} \frac{d z_{1} d z_{2}\left(z_{1}\right)^{x}\left(z_{2}\right)^{y}}{\left(1-z_{1} z_{2}\right)} C^{-1}\left(z_{1}\right) C^{-1}\left(z_{2}\right) .
$$

Представим произведение $\widehat{C} \cdot \widehat{C}^{T}$ в виде

$$
\begin{aligned}
\left(\widehat{C} \cdot \widehat{C}^{T}\right)_{x, y} & =\oint_{|z|<|\xi|<1} \frac{d(z \xi)}{z \xi} z^{-x} \xi^{y} C(z) C\left(\xi^{-1}\right) \sum_{x^{\prime}=0}^{r}\left(\frac{z}{\xi}\right)^{x^{\prime}}= \\
& =\delta_{x, y}-A_{x, y}-B_{x, y}
\end{aligned}
$$

где

$$
\begin{aligned}
& A_{x, y}=-\oint_{|\xi|<1<|z|} \frac{d(z \xi) z^{-x} \xi^{y}}{z \xi\left(1-\frac{z}{\xi}\right)} \frac{C(z)}{C(\xi)}=\oint \frac{d(z \xi) z^{x} \xi^{y}}{1-z \xi} \frac{1}{C(z) C(\xi)} \\
& B_{x, y}=\oint_{|z|<|\xi|<1} \frac{d(z \xi) z^{r+1-x} \xi^{y-r-1}}{z \xi\left(1-\frac{z}{\xi}\right)} \frac{C(z)}{C(\xi)}=\oint \frac{d(z \xi) z^{r+1-x} \xi^{y-r-1}}{1-z \xi} C(z) C(\xi) .
\end{aligned}
$$

При выводе (П.3) мы вьполнили суммирование по $x^{\prime}$ геометрической прогрессии и использовали соотношение $C\left(z^{-1}\right)=C^{-1}(z)$. Подставляя (П.3) в (П.1), получаем

$$
g(r)=\left|1-s^{2}(\hat{A}+\widehat{B})\right|=\left|\left(1-s^{2} \hat{A}\right) \cdot\left(1-s^{2} \widehat{B}\right)-s^{4} \hat{A} \cdot \widehat{B}\right|,
$$

где $s=\sin \pi \nu^{\prime}$.

Заметим, что матричные элементы матриц $\hat{A}$ и $\widehat{B}$ имеют следуюшее асимптотическое поведение:

$$
A_{x, y} \underset{x+y \gg 1}{\sim} \alpha^{x+y}, \quad B_{x, y} \underset{2 r-x-y \gg 1}{\sim} \alpha^{2 r-x-y},
$$

следовательно, произведение $\hat{A} \cdot \widehat{B}$ является экспоненциально малой матрицей. Действительно,

$$
\begin{aligned}
(\hat{A} \cdot \widehat{B})_{x y} & =\oint \frac{d\left(z_{1} \ldots z_{4}\right)}{\left(1-z_{1} z_{2}\right)\left(1-z_{3} z_{4}\right)} \frac{C\left(z_{3}\right) C\left(z_{4}\right)}{C\left(z_{1}\right) C\left(z_{2}\right)}\left(z_{1}\right)^{x}\left(z_{4}\right)^{r-y} \frac{\left(z_{3}\right)^{r+1}-\left(z_{2}\right)^{r+1}}{z_{3}\left(1-\frac{z_{2}}{z_{3}}\right)} \underset{r \rightarrow \infty}{\sim} \\
& \stackrel{\sim}{\sim} \frac{\alpha^{r} \ln r}{r}\left(\oint \frac{d z z^{x}}{1-\alpha z} C\left(z^{-1}\right)\right)\left(\oint \frac{d z z^{r-y}}{1-\alpha z} C(z)\right) .
\end{aligned}
$$


С учетом (П.5) в пределе $r \rightarrow \infty$ определитель (П.4) записывается в виде произведения определителей

$$
g(r) \underset{r \rightarrow \infty}{=}\left|\left(1-s^{2} \hat{A}\right)\right|\left|\left(1-s^{2} \widehat{B}\right)\right| .
$$

Покажем также, что $\left|\left(1-s^{2} \hat{A}\right)\right|=\left|\left(1-s^{2} \widehat{B}\right)\right|$ при $r \rightarrow \infty$. Для этого матрицу $\widehat{B}$ представим в виде

$$
\begin{gathered}
\widehat{B}=\hat{J} \cdot \widehat{D} \cdot \hat{J}, \quad J_{x, y}=\delta_{x+y, r}, \quad(\hat{J} \cdot \hat{J})_{x, y}=\delta_{x, y}, \\
D_{x, y}=\oint \frac{d(z \xi) z^{x} \xi^{y}}{1-z \xi} C(z) C(\xi) .
\end{gathered}
$$

Используя это представление, нетрудно показать, что

$$
\left|\left(1-s^{2} \hat{A}\right)\right|=\left|\left(1-s^{2} \widehat{D}\right)\right|
$$

Детерминанты в (П.8) удобно записать в виде следуюших рядов:

$$
\begin{aligned}
& \ln \left|1-s^{2} \hat{A}\right|=\operatorname{Sp} \ln \left(1-s^{2} \hat{A}\right)=-\sum_{k=1}^{\infty} \frac{s^{2 k}}{k} a_{2 k}, \\
& \ln \left|1-s^{2} \widehat{D}\right|=\operatorname{Sp} \ln \left(1-s^{2} \widehat{D}\right)=-\sum_{k=1}^{\infty} \frac{s^{2 k}}{k} d_{2 k},
\end{aligned}
$$

где

$$
a_{2 k}=\operatorname{Sp}(\hat{A})^{k}, \quad d_{2 k}=\operatorname{Sp}(\widehat{D})^{k} .
$$

В пределе $r \rightarrow \infty$ матрицы $\hat{A}$ и $\widehat{D}$ можно представить в факторизованном виде

$$
\begin{aligned}
& \hat{A} \underset{r \rightarrow \infty}{=} \widehat{F}^{(a)} \cdot \widehat{F}^{(a)}, \quad \widehat{D} \underset{r \rightarrow \infty}{=} \widehat{F}^{(d)} \cdot \widehat{F}^{(d)}, \\
& F_{x, y}^{(a)}=\oint d z z^{x+y} C(z), \quad F_{x, y}^{(d)}=\oint d z z^{x+y} C^{-1}(z),
\end{aligned}
$$

так что

$$
\begin{array}{ll}
a_{2 k}=\operatorname{Sp}\left(\widehat{F}^{(a)}\right)^{2 k}=\oint \prod_{l=1}^{2 k}\left(\frac{d z_{l} C^{-1}\left(z_{l}\right)}{1-z_{l} z_{l+1}}\right), & z_{2 k+1} \equiv z_{1}, \\
d_{2 k}=\operatorname{Sp}\left(\widehat{F}^{(d)}\right)^{2 k}=\oint \prod_{l=1}^{2 k}\left(\frac{d z_{l} C\left(z_{l}\right)}{1-z_{l} z_{l+1}}\right), & z_{2 k+1} \equiv z_{1} .
\end{array}
$$

Рассмотрим для примера случай $k=1$ :

$$
\begin{aligned}
d_{2} & =\oint_{\left|z_{1} z_{2}\right|<1} \frac{d z_{1} d z_{2}}{\left(1-z_{1} z_{2}\right)^{2}} C\left(z_{1}\right) C\left(z_{2}\right)=\oint_{\left|z_{1}\right|<\left|z_{2}\right|} \frac{d z_{1} d z_{2}}{\left(z_{1}-z_{2}\right)^{2}} \frac{C\left(z_{1}\right)}{C\left(z_{2}\right)}= \\
& =\oint_{\left|z_{2}\right|<\left|z_{1}\right|} \frac{d z_{1} d z_{2}}{\left(z_{1}-z_{2}\right)^{2}} \frac{C\left(z_{1}\right)}{C\left(z_{2}\right)}+\oint d z C(z) \frac{\partial}{\partial z} C^{-1}(z)
\end{aligned}
$$


Второе слагаемое в правой части (П.10) обращается в нуль, т.к. является интегралом по замкнутому контуру от производной, и замена переменной $z_{1} \rightarrow\left(z_{1}\right)^{-1}$ в первом слагаемом в правой части (П.10) дает

$$
d_{2}=\oint_{\left|z_{1} z_{2}\right|<1} \frac{d z_{1} d z_{2}}{\left(1-z_{1} z_{2}\right)^{2}} \frac{1}{C\left(z_{1}\right) C\left(z_{2}\right)}=a_{2} .
$$

Для $k>1$ нетрудно вывести рекуррентное соотношение

$$
d_{2 k}=a_{2 k}+d_{2 k-2}-a_{2 k-2}
$$

Для этого в выражении (П.9) для $d_{2}$ в интеграле делается замена переменных $z_{l} \rightarrow$ $\left(z_{l}\right)^{-1}$. При этом контуры интегрирования переходят в такие, что $\left|z_{l} z_{l+1}\right|>1$. Стягивая эти контуры последовательно по переменным $z_{l}$ таким образом, чтобы $\left|z_{l} z_{l+1}\right|<1$, и следя за вычетами в полюсах при $z_{l}=\left(z_{l+1}\right)^{-1}$, мы получаем соотношение (П.12). С учетом "начального условия" (П.11) решением рекуррентного соотношения (П.12) является $a_{2 k}=d_{2 k}$, из которого следует равенство (П.8). В свою очередь, соотношения (П.8) и (П.6) приводят к выполнению представления (П.1).

Заметим, что, положив в (П.2) $\mu=1 / 2$ и $\nu^{\prime}=\nu$, из (П.1) получаем (3.3), а при $\mu=\nu$ и $\nu^{\prime}=1 / 2$ из (П.1) следует (3.17).

\section{Список литературы}

[1] V.E. Korepin, N. A. Slavnov. The determinant representation for quantum correlation functions of the sinh-Gordon model. hep-th/9801046.

[2] V.E. Korepin, T. Oota. The determinant representation for quantum correlation functions in scaling Lee-Yang model. hep-th/9802003.

[3] F. A. Smirnov. Form Factors in Completely Integrable Models of Quantum Field Theory. Singapore: World Scientific, 1992.

[4] S. Lukyanov. Mod. Phys. Lett. A. 1997. V. 12. P. 2543.

[5] V. Brazhnikov, S. Lukyanov. Nucl. Phys. B. 1998. V. 512. P. 616.

[6] V. E. Korepin. Commun. Math. Phys. 1987. V. 113. P. 177.

[7] Н. А. Славнов. Зап. научн. семин. ПОМИ. 1997. Т. 245. С. 270.

[8] V.E. Korepin, N.M. Bogoliubov, A. G. Izergin. Quantum Inverse Scattering Method and Correlation Functions. Cambridge: University Press, 1993.

[9] J. B. Zuber, C. Itzykson. Phys. Rev. D. 1975. V. 11. P. 2875.

[10] B. Schroer, T. T. Truong. Nucl. Phys. B. 1978. V. 144. P. 80.

[11] P. Ginsparg. Applied conformal field theory. In: Champs, Cordes et Phenomenes Critiques. Les Houches 1988 Summer School. Eds. E. Brezin, J. Zinn-Justin. Amsterdam: North Holland, 1989. P. 1.

[12] M. Sato, T. Miwa, M. Jimbo. Publ. RIMS Kyoto Univ. 1979. V. 15. P. 871.

[13] D. Bernard, A. LeClair. Nucl. Phys. B. 1994. V. 426. P. 534.

[14] S. Lukyanov, A. Zamolodchikov. Nucl. Phys. B. 1997. V. 493. P. 571.

[15] E. L. Basor, C. A. Tracy. Inter. J. Mod. Phys. A. 1992. V. 7. Sup. 1A. P. 83.

[16] E. C. Marino, B. Schroer, J. A. Swieca. Nucl. Phys. B. 1982. V. 200. P. 473.

[17] А. И. Бугрий, В. Н. Шадура. ТМФ. 1995. Т. 103. С. 388.

[18] B. M. McCoy, T. T. Wu. The Two-Dimensional Ising Model. Cambridge: Harvard University Press, 1973. 